\title{
Stueckelberg breaking of Weyl conformal geometry and applications to gravity
}

\author{
D. M. Ghilencea* \\ Department of Theoretical Physics, National Institute of Physics and Nuclear Engineering, \\ Bucharest 077125, Romania
}

(Received 3 May 2019; accepted 22 January 2020; published 13 February 2020)

\begin{abstract}
Weyl conformal geometry may play a role in early cosmology where effective theory at short distances becomes conformal. Weyl conformal geometry also has a built-in geometric Stueckelberg mechanism: it is broken spontaneously to Riemannian geometry after a particular Weyl gauge transformation (of gauge fixing) while the Stueckelberg mechanism rearranges the degrees of freedom, conserving their number $\left(n_{d f}\right)$. The Weyl gauge field $\left(\omega_{\mu}\right)$ of local scale transformations acquires a mass after absorbing a compensator (dilaton), decouples, and Weyl connection becomes Riemannian. Mass generation has thus a dynamic origin, corresponding to a transition from Weyl to Riemannian geometry. In applications, we show that a gauge fixing symmetry transformation of the original Weyl's quadratic gravity action immediately gives the Einstein-Proca action for the Weyl gauge field and a positive cosmological constant, plus matter action (if present). As a result, the Planck scale is an emergent scale, where Weyl gauge symmetry is spontaneously broken and Einstein action is a broken phase of Weyl action. This is in contrast to local scale invariant models (no gauging) where a negative kinetic term (ghost dilaton) remains present and $n_{d f}$ is not conserved when this symmetry is broken. The mass of $\omega_{\mu}$, setting the nonmetricity scale, can be much smaller than $M_{\text {Planck }}$, for ultraweak values of the coupling $(q)$, with implications for phenomenology. If matter is present, a positive contribution to the Planck scale from a scalar field $\left(\phi_{1}\right)$ VEV(vacuum expectation value) induces a negative (mass) ${ }^{2}$ term for $\phi_{1}$ and spontaneous breaking of the symmetry under which it is charged. These results are immediate when using Weyl-covariant (invariant) scalar (tensor) curvatures, respectively, instead of their Riemannian form. Briefly, Weyl gauge symmetry is physically relevant and its role in high scale physics should be reconsidered.
\end{abstract}

DOI: $10.1103 /$ PhysRevD.101.045010

\section{WEYL GAUGE TRANSFORMATIONS AND STUECKELBERG MECHANISM}

In 1918 Weyl introduced his vector-tensor theory of quadratic gravity [1-3] built on what is now known as Weyl conformal geometry. Weyl's idea was that the action should be invariant under a most general symmetry: a Weyl scaling gauge symmetry [4]. Weyl also thought of identifying this gauge field $\left(\omega_{\mu}\right)$ with electromagnetism, which inevitably failed since electromagnetic gauge transformations are "internal" symmetry (not spacetime geometry) transformations. Weyl quadratic gravity was disregarded after Einstein's early criticism [1] that the spacing of atomic spectral lines changes in such a theory, in contrast with experience. This happens because in Weyl geometry a

\footnotetext{
*dumitru.ghilencea@cern.ch
}

Published by the American Physical Society under the terms of the Creative Commons Attribution 4.0 International license. Further distribution of this work must maintain attribution to the author(s) and the published article's title, journal citation, and DOI. Funded by SCOAP ${ }^{3}$. vector parallel transported around a curve changes not only the direction (as in Riemannian geometry) but also its length. Then clock's rates and rod's lengths depend on their path history. This is caused by the massless Weyl gauge field $\omega_{\mu}$ responsible for the nonmetric connection of Weyl geometry, $\tilde{\nabla}_{\mu} g_{\alpha \beta}=-\omega_{\mu} g_{\alpha \beta}$. This is in contrast to the Riemannian case (of $\omega_{\mu}=0$ ) and Einstein gravity where $\nabla_{\mu} g_{\alpha \beta}=0$ with $\nabla_{\mu}$ the Levi-Civita connection. Eventually (gauged) local scale transformations were abandoned and replaced by phase transformations [5] setting the foundation of modern gauge theories.

Dirac revived Weyl gravity by introducing a different version of it [6] linear in Weyl scalar curvature $(\tilde{R})$ of the form $\phi^{2} \tilde{R}$ with an additional matter scalar $\phi$ [7-18]. This term recovers Einstein gravity, and the Weyl field becomes massive (mass $\sim q M_{\text {Planck }}$ ) and decouples $\left(\omega_{\mu}=0\right.$ ); as a result, the Weyl connection becomes Riemannian and Einstein's criticism is avoided.

Recently it was shown [19] that even the original Weyl quadratic gravity without matter [1-3] avoids Einstein's criticism since $\omega_{\mu}$ again becomes massive and decouples. 
Here we explore further the consequences of this work. The result in [19] underlines the less known fact that some theories based on Weyl geometry have a built-in geometric Stueckelberg mass mechanism [20-22]. To see this more easily, we use the curvature scalar and tensors of Weyl geometry (hereafter "Weyl formulation") instead of their Riemannian expression.

The main results of this work show the following:

(a) In Weyl formulation, a simple Weyl symmetry transformation (of "gauge fixing") applied to an action in Weyl geometry gives an action directly in the Riemannian geometry with a Stueckelberg breaking of the Weyl gauge symmetry. For example, the original Weyl quadratic gravity action is "gauge transformed" into Einstein-Proca action for $\omega_{\mu}$, a cosmological constant plus matter action (if present). As a result, Einstein action is simply a spontaneously broken phase of Weyl quadratic gravity action.

(b) We stress that only gauge transformations are used in step (a), with no field re-definitions.

(c) Note that no ghost is generated and the number $n_{d f}$ of degrees of freedom (d.o.f.) (other than graviton) is conserved: the real dilaton (spin 0 mode in the $\tilde{R}^{2}$ term) is absorbed by $\omega_{\mu}$ which becomes massive; so $n_{d f}=3$ is unchanged, as expected for a spontaneous breaking. This is different from gauge fixing in conformal models (e.g., [23]) where the Stueckelberg mechanism is not available (since there is no $\omega_{\mu}$ ) so $n_{d f}$ is not conserved and a ghost dilaton is present.

(d) The Planck scale is an emergent scale where the Weyl gauge symmetry is broken.

The nonmetricity scale is set by the Weyl "photon" mass $\left(\sim q M_{\text {Planck }}\right)$, naively expected to be large. Interestingly, small values of this mass are allowed (demanding ultraweak values of its coupling $q$ ) because the lower bound on the nonmetricity scale is $\mathcal{O}(\mathrm{TeV})$ [24]. Then the Weyl field could even be a $(\mathrm{TeV})$ dark matter candidate [25]. The phenomenology of the Standard Model (SM) endowed with the Weyl gauge symmetry $[14,17]$ deserves careful study.

\section{A. Weyl gauge transformations}

Consider a Weyl scaling gauge transformation $\Omega(x)$ of the metric $g_{\mu \nu}$ and of scalar field $\phi$,

$$
\hat{g}_{\mu \nu}=\Omega g_{\mu \nu}, \quad \hat{\phi}=\frac{1}{\sqrt{\Omega}} \phi, \quad \hat{\omega}_{\mu}=\omega_{\mu}-\partial_{\mu} \ln \Omega .
$$

Here $\omega_{\mu}$ is the Weyl gauge field; we also have $\sqrt{\hat{g}}=\Omega^{2} \sqrt{g}$, $g \equiv\left|\operatorname{det} g_{\mu \nu}\right|$, and metric $(+,-,-,-)$ and conventions as in [26]. The Weyl-covariant derivative of $\phi$ is

$$
\begin{aligned}
\tilde{D}_{\mu} \phi & =\left(\partial_{\mu}-1 / 2 \omega_{\mu}\right) \phi \\
& =(-1 / 2) \phi\left[\omega_{\mu}-\partial_{\mu} \ln \phi^{2}\right] .
\end{aligned}
$$

$\Omega(x)$ is real, and there is no complex factor " $i$ " in (1) or in $\tilde{D}_{\mu} \phi$. The gauge symmetry is a dilatation group that is isomorphic to $R^{+}$. $\tilde{D}_{\mu} \phi$ transforms under (1) as a scalar field $\hat{\tilde{D}}_{\mu} \hat{\phi}=(1 / \sqrt{\Omega}) \tilde{D}_{\mu} \phi$. Given (1), $\omega_{\mu}$ has a geometric origin while Eq. (3) has an obvious resemblance to the Stueckelberg mechanism; see later.

In Weyl geometry $\left(\tilde{\nabla}_{\mu}+\omega_{\mu}\right) g_{\alpha \beta}=0$, with $\tilde{\nabla}_{\mu}$ defined by the Weyl connection coefficients denoted $\tilde{\Gamma}_{\mu \nu}^{\rho}$. This differs from Riemannian geometry where $\nabla_{\mu} g_{\alpha \beta}=0$ with $\nabla_{\mu}$ defined by the Levi-Civita connection $\Gamma_{\mu \nu}^{\rho}=$ $(1 / 2) g^{\rho \beta}\left(\partial_{\nu} g_{\beta \mu}+\partial_{\mu} g_{\beta \nu}-\partial_{\beta} g_{\mu \nu}\right) . \tilde{\Gamma}_{\mu \nu}^{\rho}$ can be found from $\Gamma_{\mu \nu}^{\rho}$ by replacing $\partial_{\mu} \rightarrow \partial_{\mu}+\omega_{\mu}$ and giving $\tilde{\Gamma}_{\mu \nu}^{\rho}=\Gamma_{\mu \nu}^{\rho}+$ $(1 / 2)\left[\delta_{\mu}^{\rho} \omega_{\nu}+\delta_{\nu}^{\rho} \omega_{\mu}-g_{\mu \nu} \omega^{\rho}\right]$.

$\tilde{\Gamma}_{\mu \nu}^{\rho}$ are symmetric $\left(\tilde{\Gamma}_{\mu \nu}^{\rho}=\tilde{\Gamma}_{\nu \mu}^{\rho}\right)$ (no torsion) and are invariant under (1) since their variation induced by the metric is compensated by that of $\omega_{\mu}$. The Riemann and Ricci tensors in Weyl geometry are defined as in Riemannian geometry but in terms of new $\tilde{\Gamma}_{\mu \nu}^{\rho}$, and are also invariant under (1). ${ }^{1}$ One can then show that the Weyl scalar curvature $(\tilde{R})$

$$
\tilde{R}=R-3 D_{\mu} \omega^{\mu}-\frac{3}{2} \omega^{\mu} \omega_{\mu},
$$

where $R$ is the Riemannian scalar curvature and $D_{\mu} \omega^{\mu}$ is defined by the Levi-Civita connection.

Using the curvature tensors and scalar of Weyl geometry has an advantage: unlike in the Riemannian case, $\widetilde{R}$ transforms covariantly under (1) similar to $g^{\mu \nu}$ entering its definition:

$$
\hat{\tilde{R}}=\frac{1}{\Omega} \tilde{R}
$$

This simplifies our calculations and helps build Weyl gauge invariant individual operators. Then this symmetry and internal gauge symmetries are on an equal footing in the action.

The criticisms of Weyl gravity based on Weyl geometry (such as the change of a vector length under parallel displacement or of atomic spectral lines spacing) are avoided if $\omega_{\mu}=0$ because from above $\tilde{\Gamma}_{\mu \nu}^{\rho}=\Gamma_{\mu \nu}^{\rho}$, $\tilde{R}=R$, Weyl connection becomes Levi-Civita, the geometry becomes Riemannian, and these criticisms do not apply. This happens if we do not introduce $\omega_{\mu}$ in (1); i.e., we go back to Riemannian geometry gravity (e.g., BransDicke). Alternatively, $\omega_{\mu}=0$ after this field acquires a large mass and decouples. This is the idea we study below.

\footnotetext{
${ }^{1}$ These $\quad$ are $\tilde{R}_{\mu \nu \sigma}^{\lambda}=\partial_{\nu} \tilde{\Gamma}_{\mu \sigma}^{\lambda}-\partial_{\sigma} \tilde{\Gamma}_{\mu \nu}^{\lambda}+\tilde{\Gamma}_{\nu \rho}^{\lambda} \tilde{\Gamma}_{\mu \sigma}^{\rho}-\tilde{\Gamma}_{\sigma \rho}^{\lambda} \tilde{\Gamma}_{\mu \nu}^{\rho}$ and $\tilde{R}_{\mu \sigma}=\tilde{R}_{\mu \lambda \sigma}^{\lambda}$. Also we have $\tilde{R}=g^{\mu \sigma} \tilde{R}_{\mu \sigma}$.
} 


\section{B. Weyl gauge transformation and Proca action}

Consider $L$ of a real scalar field $\phi$ with coupling $q$ to a Weyl gauge field $\omega_{\mu}$, invariant under (1)

$$
L=\sqrt{g}\left[-\frac{1}{4 q^{2}} F_{\mu \nu}^{2}+\frac{1}{2}\left(\tilde{D}_{\mu} \phi\right)^{2}\right] .
$$

To simplify notation, we do not show appropriate indices contractions which are implicit, e.g., $F_{\mu \nu}^{2}=g^{\mu \nu} g^{\rho \sigma} F_{\mu \rho} F_{\nu \sigma}$ and $\left(\tilde{D}_{\mu} \phi\right)^{2}=g^{\mu \nu} \tilde{D}_{\mu} \phi \tilde{D}_{\nu} \phi$. Since there is no torsion, the field strength $F_{\mu \nu}$ does not feel the connection. From $F_{\mu \nu}=$ $\tilde{D}_{\mu} \omega_{\nu}-\tilde{D}_{\nu} \omega_{\mu}$ with $\tilde{D}_{\mu} \omega_{\nu}=\partial_{\mu} \omega_{\nu}-\tilde{\Gamma}_{\mu \nu}^{\rho} \omega_{\rho}$ then $F_{\mu \nu}=\partial_{\mu} \omega_{\nu}-$ $\partial_{\nu} \omega_{\mu}$, which coincides with its Riemannian expression and is invariant under (1). A gauge transformation (1) with $\Omega=\phi^{2} / M^{2}$ gives

$$
L=\sqrt{\hat{g}}\left[-\frac{1}{4 q^{2}} \hat{F}_{\mu \nu}^{2}+\frac{1}{8} M^{2} \hat{\omega}_{\mu} \hat{\omega}^{\mu}\right],
$$

where $M$ is an arbitrary scale and all indices contractions are made with a new metric $\left(\hat{g}_{\mu \nu}\right)$.

The Weyl "photon" has become massive and no trace of $\phi$ is left; see [22] for a more general discussion. This is a geometric version of the Stueckelberg mechanism [20,21] that is naturally built-in Weyl conformal geometry due to the definition of Weyl-covariant derivative $\tilde{D}_{\mu}$. The presence of $\sqrt{g}$ is essential as it ensures invariance of $L$. The (charged) scalar and Weyl kinetic term are gauge transformed into an equivalent Proca action with spontaneous breaking of the Weyl gauge symmetry. If we do the inverse gauge transformation, Proca action of a massive theory can be written in a Weyl gauge invariant way as a sum of kinetic terms.

The gauge transformation we did is essentially gauge fixing $\phi=M$ (constant) but what is most important here is the conservation of the number of dynamical d.o.f., $n_{d f}$ $\left(n_{d f}=3\right)$ : initially we had a massless scalar and a massless vector field and finally a massive vector field. $q M$ is regarded as the scale where Weyl gauge symmetry is broken.

If in (6) there are more $(n)$ scalar fields kinetic terms, consider a gauge transformation $\Omega=\rho^{2} / M^{2}$, with $\rho$ the radial direction $\rho^{2}=\sum_{j} \phi_{j}^{2}$, absorbed by the only vector field present $\hat{\omega}_{\mu}=\omega_{\mu}-\partial_{\mu} \ln \rho^{2}$ under (1). One recovers (7) with $n-1$ additional kinetic terms for the angular variables fields. To conclude, the Weyl boson is massive and can decouple.

\section{Weyl linear gravity as Einstein-Proca action}

Consider a linear version of Weyl gravity [6] coupled to a scalar $\phi_{1}$, invariant under (1),

$\mathcal{L}=\sqrt{g}\left[-\frac{\xi_{1}}{12} \phi_{1}^{2} \tilde{R}+\frac{1}{2} g^{\mu \nu} \tilde{D}_{\mu} \phi_{1} \tilde{D}_{\nu} \phi_{1}-\frac{\lambda_{1}}{4 !} \phi_{1}^{4}-\frac{1}{4 q^{2}} F_{\mu \nu}^{2}\right]$, where $\tilde{R}$ is the scalar curvature in Weyl geometry, Eq. (4), and $\tilde{D}_{\mu} \phi_{1}=\left(\partial_{\mu}-1 / 2 \omega_{\mu}\right) \phi_{1}$.

After a Weyl gauge transformation (1), (5), with $\Omega=\xi_{1} \phi_{1}^{2} /\left(6 M^{2}\right)$, and then using Eq. (4),

$$
\begin{aligned}
\mathcal{L}= & \sqrt{\hat{g}}\left[-\frac{1}{2} M^{2} \hat{R}-\frac{1}{4 q^{2}} \hat{F}_{\mu \nu}^{2}+\frac{3}{4} M^{2}\left(1+1 / \xi_{1}\right) \hat{\omega}_{\mu} \hat{\omega}^{\mu}\right. \\
& \left.-\frac{3 \lambda_{1} M^{4}}{2 \xi_{1}^{2}}\right],
\end{aligned}
$$

up to a total derivative term. Here Riemannian scalar curvature $\hat{R}$ and indices contractions are computed with new $\hat{g}_{\mu \nu}$, as indicated by the presence of $\sqrt{\hat{g}}$.

The gauge transformation considered sets $\hat{\phi}_{1}$ to a constant $\left(6 M^{2} / \xi_{1}\right)$, and the Einstein frame results from gauge fixing Weyl gauge symmetry. The Stueckelberg mechanism ensures the number of dynamical d.o.f. $n_{d f}$ is conserved when going from (8) to (9) as expected for spontaneous breaking (and which does not require a potential for $\phi_{1}$ ). Here $\phi_{1}$ was "eaten" by the Weyl gauge field, which is now massive. What survives of the scalar kinetic term is the $\xi$-dependent mass term for $\hat{\omega}_{\mu}$, but there is an additional mass correction to $\hat{\omega}_{\mu}$ beyond (7), due to the $\tilde{R}$-dependent term.

This situation is in contrast with the (ungauged) local conformal symmetry case recovered from (8) for $\omega_{\mu}=0$; then there is no gauge field to "absorb" the scalar "compensator" and the action would be invariant under the first two transformations in (1) only if $\xi_{1}=-1$.

To conclude, the Weyl photon again became massive by "absorbing" a compensator field $\phi_{1}$. But what happens in Weyl quadratic gravity with no matter fields present?

\section{Weyl quadratic gravity as Einstein-Proca action}

The original action of Weyl (quadratic) gravity without matter [2] invariant under (1) is

$$
L_{1}=\sqrt{g}\left[\frac{\xi_{0}}{4 !} \tilde{R}^{2}-\frac{1}{4 q^{2}} F_{\mu \nu}^{2}\right], \quad \xi_{0}>0 .
$$

Each term is Weyl gauge invariant $[\tilde{R}$ transforms covariantly, Eq. (5)]. We can replace $\tilde{R}^{2} \rightarrow-2 \phi_{0}^{2} \tilde{R}-\phi_{0}^{4}$, since integrating the auxiliary field $\phi_{0}$ via its equation of motion, of solution $\phi_{0}^{2}=-\tilde{R}$, recovers the $\tilde{R}^{2}$ term in the action; so $\phi_{0}$ transforms as any scalar field and $\ln \phi_{0}$ is the Goldstone of the scale symmetry (1), $\ln \phi_{0}^{2} \rightarrow \ln \phi_{0}^{2}-\Omega$. Then

$$
L_{1}=\sqrt{g}\left[\frac{\xi_{0}}{4 !}\left(-2 \phi_{0}^{2} \tilde{R}-\phi_{0}^{4}\right)-\frac{1}{4 q^{2}} F_{\mu \nu}^{2}\right]
$$

Using gauge transformation (1), (5) with $\Omega=\xi_{0} \phi_{0}^{2}$ / $\left(6 M^{2}\right)$, and then using relation (4) we find 
$L_{1}=\sqrt{\hat{g}}\left\{-\frac{1}{2} M^{2} \hat{R}-\frac{3 M^{4}}{2 \xi_{0}}+\frac{3}{4} M^{2} \hat{\omega}_{\mu} \hat{\omega}^{\mu}-\frac{1}{4 q^{2}} \hat{F}_{\mu \nu}^{2}\right\}$,

which is in the Einstein frame. Here we chose $M=M_{\text {Planck }}$ ( $\hat{R}$ is the Riemannian scalar curvature evaluated from new metric $\hat{g}_{\mu \nu}$ also used for index contractions).

These simple steps show an interesting result: a Weyl gauge fixing symmetry transformation (not fields redefinition) applied to the original Weyl quadratic gravity without matter Eq. (10) gives the Einstein-Proca action for the Weyl gauge field; this became massive via the Stueckelberg mechanism (spontaneous breaking). There is also a positive cosmological constant. Conversely, the inverse gauge transformation of Einstein-Proca action takes one to Weyl quadratic gravity action. Note again the conservation of the number of d.o.f., impossible in the absence of the Weyl gauge field.

To illustrate better the Stueckelberg mechanism, write first Eq. (11) in a Riemannian language using Eq. (4) followed by an integration by parts, which gives

$$
\begin{aligned}
L_{1}= & \sqrt{g}\left\{-\frac{\xi_{0}}{2}\left[\frac{1}{6} \phi_{0}^{2} R+\left(\partial_{\mu} \phi_{0}\right)^{2}\right]-\frac{\xi_{0}}{4 !} \phi_{0}^{4}\right. \\
& \left.+\frac{1}{8} \xi_{0} \phi_{0}^{2}\left(\omega_{\mu}-\partial_{\mu} \ln \phi_{0}^{2}\right)^{2}-\frac{1}{4 q^{2}} F_{\mu \nu}^{2}\right\},
\end{aligned}
$$

where we used that $\sqrt{g} D_{\mu} \omega^{\mu}=\partial_{\mu}\left(\sqrt{g} \omega^{\mu}\right)$. Then using gauge transformation (1) with $\Omega=\xi_{0} \phi_{0}^{2} /\left(6 M^{2}\right)$, one finds again Eq. (12). It is then obvious how the first term becomes the Einstein term in (12) and how the term of coefficient $1 / 8$ gives the mass term for $\hat{\omega}_{\mu}$ (Stueckelberg mechanism) in (12). Note there is no negative kinetic term (ghost) in Eq. (13).

The mass of the Weyl gauge boson is near the Planck scale $(\sqrt{3 / 2} q M)$ for a coupling $q$ not too small and comes from the $\tilde{R}^{2}$ term alone. Below this mass scale this field decouples, the Weyl connection becomes Riemannian $\left(\omega_{\mu}=0\right)$, and the Weyl quadratic action becomes Einstein-Hilbert action. So Einstein gravity is just a "low energy" limit (broken phase) of Weyl gravity. Then previous, long-held criticisms of Weyl quadratic gravity are avoided; the effects mentioned earlier, associated with Weyl geometry, are suppressed by a large value of the Weyl "photon" mass $\propto$ Planck scale. Then any change of the spacing of the atomic spectral lines is suppressed by this high scale and can be safely ignored.

The result in (12) is in the Einstein gauge of constant $\phi_{0}^{2}=6 M^{2} / \xi_{0}$ which coincides with the Weyl gauge (of constant Weyl scalar curvature) since we saw $\left\langle\phi_{0}\right\rangle^{2}=-\tilde{R}$, so on the ground state $\phi_{0}^{2}=(-\tilde{R})=6 M^{2} / \xi_{0}$; see also [10] for a discussion. Actually, for a Friedmann-RobertsonWalker universe, the scalar field naturally evolves in time to $\phi_{0}=$ const because of a conserved current $J_{\mu}=\phi_{0} \partial_{\mu} \phi_{0}$ [27]. The Planck scale thus emerges naturally as the scale where Weyl gauge symmetry is spontaneously broken.

\section{E. A more general case}

In a most general case, Weyl quadratic gravity can contain another independent term ${ }^{2,3}$

$$
\begin{aligned}
L_{1}^{\prime} & =\sqrt{g}\left\{\frac{1}{\eta} \tilde{C}_{\mu \nu \rho \sigma} \tilde{C}^{m u \nu \rho \sigma}+\frac{\xi_{0}}{4 !} \tilde{R}^{2}\right\} \\
& =\sqrt{g}\left\{\frac{1}{\eta}\left[C_{\mu \nu \rho \sigma} C^{\mu \nu \rho \sigma}+\frac{3}{2} F_{\mu \nu}^{2}\right]+\frac{\xi_{0}}{4 !} \tilde{R}^{2}\right\},
\end{aligned}
$$

where $\tilde{C}_{\mu \nu \rho \sigma}$ and $\left(C_{\mu \nu \rho \sigma}\right)$ is the Weyl tensor in Weyl geometry (Riemannian geometry), respectively; these tensors are related as shown above [16] with $F_{\mu \nu}$ the field strength of the Weyl gauge boson. Notice that in this case the $F_{\mu \nu}^{2}$ term is automatically present, so there is no need to add it "by hand" [on symmetry grounds as in (10)]; however, for a canonical gauge kinetic term one has in this case $q^{2}=-\eta / 6(\eta<0)$. The result of Eq. (12) is still valid since both Weyl tensors are invariant under Weyl gauge transformations; then the final Lagrangian contains an additional term $C_{\mu \nu \rho \sigma}^{2}$; this is needed anyway at the quantum level when trying to renormalize $\mathrm{SM}$ in the presence of gravity in (ungauged) local conformal models [23]. In this case and in the absence of a separate kinetic term for $\omega_{\mu}$ in the first line of (14) (allowed by the symmetry), the mass of the Weyl gauge field $m_{\omega}^{2} \sim q^{2} M^{2} \sim$ $(-\eta) M^{2}$ is thus related to the mass of the spin-two ghost contained in $C_{\mu \nu \rho \sigma}^{2}$. At this scale the nonmetricity of Weyl geometry steps in to modify the Levi-Civita connection.

\section{F. Adding matter}

Consider now Weyl quadratic gravity, Eq. (10), coupled to a matter scalar $\phi_{1}$,

$$
\begin{aligned}
L_{2}= & \sqrt{g}\left[\frac{\xi_{0}}{4 !} \tilde{R}^{2}-\frac{1}{4 q^{2}} F_{\mu \nu}^{2}\right]-\frac{\sqrt{g}}{12} \xi_{1} \phi_{1}^{2} \tilde{R} \\
& +\sqrt{g}\left[\frac{1}{2} g^{\mu \nu} \tilde{D}_{\mu} \phi_{1} \tilde{D}_{\nu} \phi_{1}-\frac{\lambda_{1}}{4 !} \phi_{1}^{4}\right],
\end{aligned}
$$

which is invariant under (1) and the potential for $\phi_{1}$ is the only one allowed by this symmetry.

As in Eq. (11), replace $\widetilde{R}^{2} \rightarrow-2 \phi_{0}^{2} \tilde{R}-\phi_{0}^{4}$, to obtain a classically equivalent action

\footnotetext{
${ }^{2}$ A Gauss-Bonnet (total derivative) term of Weyl geometry can also be present [16], but is not relevant here.

${ }^{3}$ The Weyl tensor squared term we included here is usually required at the quantum level.
} 


$$
L_{2}=\sqrt{g}\left[-\frac{1}{2} \rho^{2} \tilde{R}-\frac{1}{4 q^{2}} F_{\mu \nu}^{2}+\frac{1}{2} g^{\mu \nu} \tilde{D}_{\mu} \phi_{1} \tilde{D}_{\nu} \phi_{1}-\mathcal{V}\left(\phi_{1}, \rho\right)\right],
$$

with

$$
\begin{aligned}
\mathcal{V}\left(\phi_{1}, \rho\right) & =\frac{1}{4 !}\left[\frac{1}{\xi_{0}}\left(6 \rho^{2}-\xi_{1} \phi_{1}^{2}\right)^{2}+\lambda_{1} \phi_{1}^{4}\right], \quad \text { with } \\
\rho^{2} & =\frac{1}{6}\left(\xi_{1} \phi_{1}^{2}+\xi_{0} \phi_{0}^{2}\right)
\end{aligned}
$$

where we replaced $\phi_{0}$ by $\rho$. Using Eq. (5), a Weyl gauge transformation (1) with $\Omega=\rho^{2} / M^{2}$ followed by (4) that introduces Riemannian $\hat{R}$ gives

$$
\begin{aligned}
L_{2}= & \sqrt{\hat{g}}\left\{-\frac{1}{2} M^{2}\left[\hat{R}-\frac{3}{2} \hat{\omega}_{\mu} \hat{\omega}^{\mu}\right]-\frac{1}{4 q^{2}} \hat{F}_{\mu \nu}^{2}\right. \\
& \left.\left.+\frac{\hat{g}^{\mu \nu}}{2} \hat{\tilde{D}}_{\mu} \hat{\phi}_{1} \hat{\tilde{D}}_{\nu} \hat{\phi}_{1}-\mathcal{V}\left(\hat{\phi}_{1}, M\right)\right]\right\},
\end{aligned}
$$

with $\hat{\tilde{D}}_{\mu} \hat{\phi}_{1}=\left(\partial_{\mu}-1 / 2 \hat{\omega}_{\mu}\right) \hat{\phi}_{1}$ and $M$ identified with $M_{\text {Planck. }}$. As in the case without matter, we obtained the Einstein-Proca action of a gauge field that became massive after the Stueckelberg mechanism of absorbing the dilaton $\ln \rho$. The mass of $\omega_{\mu}$ is $m_{\omega}^{2}=(3 / 2) q^{2} M^{2}$ [after rescaling $\hat{\omega}_{\mu} \rightarrow q \hat{\omega}_{\mu}$ in (18)]. A canonical kinetic term of $\phi_{1}$ remains, since only 1 d.o.f. (radial direction $\rho$ ) is eaten by the vector field; see Sec. IB.

Under the same gauge transformation (gauge fixing) the initial potential $\phi_{1}^{4}$ becomes

$$
\mathcal{V}=\frac{3 M^{4}}{2 \xi_{0}}\left[1-\frac{\xi_{1} \hat{\phi}_{1}^{2}}{6 M^{2}}\right]^{2}+\frac{\lambda_{1}}{4 !} \hat{\phi}_{1}^{4}
$$

We have a negative mass term $\left(m_{\hat{\phi}_{1}}^{2}=-\xi_{1} M^{2} / \xi_{0}\right)$ if $\xi_{1}>0$. This originates in (17) due to the initial dilaton contribution to the potential $\propto \phi_{0}^{4}$ (coming from $\tilde{R}^{2}$ ), with $\phi_{0}$ replaced by $\left(6 \rho^{2}-\xi_{1} \phi_{1}^{2}\right)$ and $\rho$ "gauge fixed" to $M$. Then, if massless $\phi_{1}$ gives a positive contribution $\xi_{1} \phi_{1}^{2}$ to the Planck scale $(M)\left(\xi_{1}>0\right)$ this effect is "compensated" by a negative contribution to its mass term in the potential (and vice versa) in (17). The original dilaton (in $\tilde{R}^{2}$ ) plays a mediator role in bringing this negative contribution. It is then interesting that both mass scales of the theory, the Planck scale and the scale $\left\langle\hat{\phi}_{1}\right\rangle$, are simultaneously generated by the same gauge fixing transformation (1).

If $\phi_{1}$ is the Higgs field and $\left\langle\phi_{1}\right\rangle$ is the electroweak (EW) scale, then the Stueckelberg mechanism also triggers EW breaking. This discussion remains valid for more matter fields $\phi_{j}$, and in Eqs. (15)-(19) simply replace $\xi_{1} \phi_{1}^{2} \rightarrow$ $\sum_{j} \xi_{j} \phi_{j}^{2}$ and $\left(\tilde{D}_{\mu} \phi_{1}\right)^{2} \rightarrow \sum_{j}\left(\tilde{D}_{\mu} \phi_{j}\right)^{2}$.

\section{G. Additional effects, more scalar fields, and SM in Weyl geometry}

The Weyl-covariant derivative acting on $\hat{\phi}_{1}$ in (18) is a remnant of Weyl gauge symmetry, now broken. To have a "standard" kinetic term for $\phi_{1}$, i.e., remove couplings $\hat{\omega}^{\mu} \partial_{\mu} \hat{\phi}_{1}$ (similar to electroweak "unitary gauge") one can now do a field redefinition

$\hat{\omega}_{\mu}^{\prime}=\hat{w}_{\mu}-\partial_{\mu} \ln \left(\hat{\phi}_{1}^{2}+6 M^{2}\right), \quad \hat{\phi}_{1}=M \sqrt{6} \sinh \left[\frac{\sigma}{M \sqrt{6}}\right]$

to find ${ }^{4}$

$$
\begin{aligned}
L_{2}= & \sqrt{\hat{g}}\left\{-\frac{1}{2} M^{2} \hat{R}+\frac{3}{4} M^{2} \cosh ^{2}\left[\frac{\sigma}{M \sqrt{6}}\right] \hat{\omega}_{\mu}^{\prime} \hat{\omega}^{\prime \mu}\right. \\
& \left.-\frac{1}{4 q^{2}} \hat{F}_{\mu \nu}^{\prime 2}+\frac{\hat{g}^{\mu \nu}}{2} \partial_{\mu} \sigma \partial_{\nu} \sigma-\hat{\mathcal{V}}\right\}
\end{aligned}
$$

with

$\hat{\mathcal{V}}=\frac{3}{2} \frac{M^{4}}{\xi_{0}}\left[1-\xi_{1} \sinh ^{2} \frac{\sigma}{M \sqrt{6}}\right]^{2}+\frac{3}{2} M^{4} \lambda_{1} \sinh ^{4} \frac{\sigma}{M \sqrt{6}}$.

In (21) one finally rescales $\hat{\omega}_{\mu}^{\prime} \rightarrow q \hat{\omega}_{\mu}^{\prime}$ for a canonical gauge kinetic term.

Taylor expanding the mass term of the Weyl gauge field for small $\sigma<M$ shows there are additional corrections to this mass beyond those due to the Stueckelberg mechanism, since $\langle\sigma\rangle \neq 0$. Note there is no restriction in the action regarding the relative values of $\sigma$ versus $M$. For $\sigma>M, \hat{\mathcal{V}}$ is always positive if $\xi_{1}^{2} / \xi_{0}+\lambda_{1}>0$ which can be true even for $\lambda_{1}<0$.

This potential is relevant for models of inflation, assuming $\sigma$ is the inflaton. Since Planck scale emerged as the scale where Weyl gauge symmetry is spontaneously broken, Eq. (18), field values above this scale are natural, which is relevant for inflation. For $\lambda_{1}$ and $\xi_{1}$ very small, e.g., $\lambda_{1} \xi_{1} \sim 5 \times 10^{-10}$ and $\xi_{1} \sim 10^{-5}-10^{-3}$, the potential is nearly flat and one has inflation similar to the Starobinsky model [28] for suitable $\xi_{0} \approx 10^{10}$; see [29] for the analysis of this potential. The larger quoted values of $\xi_{1}$ mark a departure from the Starobinsky inflation. But unlike in [29] where no Stueckelberg mechanism takes place since there is no gauge kinetic term, here there is no flat direction left—this was "absorbed" by the gauge field that became

\footnotetext{
${ }^{4}$ In terms of the initial fields $\omega_{\mu}$ and $\phi_{0,1}$ Eq. (20) can be written as $\hat{\omega}_{\mu}^{\prime}=\omega_{\mu}-\partial_{\mu} \ln K$ where we denoted $K \equiv \xi_{0} \phi_{0}^{2}+\left(1+\xi_{1}\right) \phi_{1}^{2}$ and there is a current $J^{\mu}=(-1 / 4) g^{\mu \nu} \nabla_{\nu} K=(-1 / 4) g^{\mu \nu}\left(\partial_{\nu}-\omega_{\nu}\right) K$, which is a total derivative and is conserved $\nabla_{\mu} J^{\mu}=0$. This is shown by applying $\partial_{\mu}$ on the equation of motion for $\omega_{\mu}$ : $q \sqrt{g} J^{\mu}+\partial_{\rho}\left(\sqrt{g} F^{\rho \mu}\right)=0$. Notice that one can also write the current as $J^{\mu}=(1 / 4) K \hat{\omega}_{\mu}^{\prime}$.
} 
massive. This issue and inflation are discussed in detail elsewhere [30].

The situation here is also different from the common models of inflation of no matter field present with inflation driven by $\sqrt{g}\left(R^{2}+M^{2} R\right)$. Here the "scalaron" mode is actually a compensator eaten by $\omega_{\mu}$, while the matter field $\sigma$ is the inflaton. Further, if there is no Weyl gauge field in (15) (set $\omega_{\mu}=0$ ), inflation is still possible and was already studied in [31]. The scenario is again similar to that in Starobinsky models. Finally, in the absence of the quadratic term, with only a linear term in scalar curvature and global scale invariance, inflation is again possible and was discussed in [27,32].

The analysis in Sec. IF can be extended to more scalar fields present in a Weyl gauge invariant action. For example, for two scalar fields in Eq. (15) with nonminimal couplings $\xi_{1}, \xi_{2}$, and with an initial potential $V\left(\phi_{1}, \phi_{2}\right)$ replacing that in (15), one obtains the following canonical action, similar to that in (21):

$$
\begin{aligned}
L_{2}= & \sqrt{\hat{g}}\left\{\frac{-1}{2} M^{2} \hat{R}-\frac{1}{4 q^{2}} \hat{F}_{\mu \nu}^{\prime} \hat{F}^{\prime \mu \nu}+\frac{1}{2} m^{2}(\sigma) \hat{\omega}_{\mu}^{\prime} \hat{\omega}^{\prime \mu}\right. \\
& \left.+\frac{1}{2}\left[\sinh ^{2} \frac{\sigma}{M \sqrt{6}}\left(\partial_{\mu} \tilde{\theta}\right)^{2}+\left(\partial_{\mu} \sigma\right)^{2}\right]-\hat{\mathcal{V}}\right\}
\end{aligned}
$$

with the notation $m^{2}(\sigma)=(3 / 4) M^{2} \cosh ^{2}(\sigma /(M \sqrt{6}))$ and with new polar coordinates fields, $\tan \theta=\phi_{1} / \phi_{2}, \phi_{1}^{2}+\phi_{2}^{2}=$ $6 M^{2} \sinh ^{2} \sigma /(M \sqrt{6})$, and $\tilde{\theta}=M \sqrt{6} \theta$. Finally, the potential is

$$
\begin{aligned}
\hat{\mathcal{V}}= & \frac{3 M^{4}}{2 \xi_{0}}\left[1-\xi_{12} \sinh ^{2} \frac{\sigma}{M \sqrt{6}}\right]^{2} \\
& +\frac{3}{2} M^{4}\left(24 V\left(s_{\theta}, c_{\theta}\right)\right) \sinh ^{4} \frac{\sigma}{M \sqrt{6}},
\end{aligned}
$$

where $\xi_{12}=\left(\xi_{1} \sin ^{2} \theta+\xi_{2} \cos ^{2} \theta\right)$ and $s_{\theta}=\sin \theta, c_{\theta}=\cos \theta$. For an $\mathcal{O}(2)$ symmetry, $\theta$ dependence in the potential disappears so one can introduce $V\left(s_{\theta}, c_{\theta}\right)=\lambda / 4$ ! but kinetic mixing remains. Compare (22) and (24) to notice the similar structure of the potential.

These results make it attractive to consider the Weyl gauge symmetry for model building beyond SM and Einstein gravity. With Einstein gravity as a low energy broken phase of Weyl quadratic gravity, then Weyl gauge symmetry and Weyl gravity are "freed" from past criticisms based on the (wrong) assumption that the Weyl gauge field is massless. One can consider the SM with a Higgs mass parameter set to zero and extend it with Weyl gauge symmetry. In such a case note that, interestingly, only the SM Higgs/scalars couple to the Weyl gauge boson, as in (15) or equivalently (21). The SM fermions do not couple to $\omega_{\mu}[14,17,33]$. The SM gauge fields kinetic terms are also invariant under Weyl gauge symmetry; see, e.g., [17]. Therefore, the SM Lagrangian formulated in Weyl conformal geometry is

$$
L=L_{2}+L_{f+g}^{\mathrm{SM}}
$$

with $L_{2}$ as in (15) adapted for the Higgs sector and other scalar fields (e.g., inflaton), as above. $L_{f+g}^{\mathrm{SM}}$ denotes the SM Lagrangian for the fermionic and gauge sectors. Note, however, that a kinetic mixing of $\omega_{\mu}$ with $U(1)_{Y}$ is allowed by the SM and by Weyl gauge symmetry. This has implications for phenomenology not yet explored. ${ }^{5}$

\section{H. Related models}

There is a difference between the Weyl gauge invariant model discussed here and the case of local conformal extensions of the Standard Model (see, e.g., [23,34,35]) when generating the Planck scale spontaneously (by dilaton VEV (vacuum expectation value)). As we saw, a Weyl gauge invariant model conserves the number of d.o.f. $n_{d f}$ during the breaking of this symmetry. Moreover, there is no ghost field in Sec. I D when gauge fixing the Planck scale in Eqs. (12) and (13). This is to be compared to these local conformal extensions of the SM where the Einstein term $(-\sqrt{g} / 2) M^{2} R$ is written in a local conformal invariant way as

$$
\mathcal{L}_{E}=\sqrt{g}\left\{-\frac{\xi_{0}}{2}\left[\frac{1}{6} \phi_{0}^{2} R+\left(\partial_{\mu} \phi_{0}\right)^{2}\right]\right\}
$$

to be compared to (13). We see here that trying to generate a Planck scale as a VEV of $\phi_{0}$, by gauge fixing $\phi_{0}^{2}=6 M^{2} / \xi_{0}$, demands the notorious negative kinetic term (ghost dilaton) be present (see [15,17] for a discussion). Also, in this local conformal case, when gauge fixing $\phi_{0}$ to a constant (unitary gauge) and this symmetry is broken, there is no gauge field to "absorb" this scalar (dilaton) mode; see, e.g., [23]. Therefore $n_{d f}$ is not conserved and shows the need for the Weyl gauge symmetry, for self-consistency.

Models with Weyl gauge symmetry seem to be allowed by black-hole physics. This is not true for models with global symmetries, in particular global scale symmetry (e.g., Agravity [36]) since global charges can be eaten by black holes that subsequently evaporate [37]. Further, in models with Weyl gauge symmetry higher dimensional/ curvature operators (beyond quadratic ones of dimension $d=4$ in the Weyl action) cannot be present since they should be suppressed by some high scale not present in the theory (forbidden by this symmetry). Also, the dilaton is eaten by the Weyl photon which becomes massive, so dilaton powers cannot be present to suppress such effective operators either. This may remain true at the quantum level, assuming quantum calculations respect this symmetry. This requires an ultraviolet regularization that preserves the Weyl gauge symmetry [38] (see also [39]).

\footnotetext{
${ }^{5}$ This mixing can be neglected for a large enough nonmetricity scale (mass $m_{\omega}$ ).
} 
This analysis can be extended to other nonmetric theories, with torsion, etc. Our result is in agreement with more general approaches [22] that consider that at a fundamental level gravity is a theory of connections as dynamical objects. Some of these connections become massive (via Stueckelberg mechanism), as we saw for the Weyl connection, while the Levi-Civita connection remains massless. In our case the Weyl connection departed from the (fixed) Levi-Civita by a correction $\omega_{\mu}$ which was a dynamical field. More generally, one can write any dynamical connection as a Levi-Civita connection plus a tensor field contribution which is a sum of a nonmetricity tensor (here due to $\omega_{\mu}$ ) and a contorsion tensor, and then redo this analysis. From a particle physics perspective, this tensor field, being massive, should decouple and leave in the low energy limit only the Levi-Civita connection.

For high scale physics and early cosmology, nonmetricity effects cannot be ignored. In fact, current lower bounds on the nonmetricity scale, which is set by the mass of the Weyl field, are very low, in the region of a few $\mathrm{TeV}$ [24]. With the mass of Weyl field $\omega_{\mu}$ of $\sqrt{3 / 2} q M_{\text {Planck }}$, this region would correspond to ultraweak values of the coupling $q$. One may also explore the possibility that $\omega_{\mu}$ is a dark matter candidate. In Weyl invariant models of vector dark matter $(\mathrm{DM})$, the mass of the $\mathrm{DM}$ vector field is again in the region of a few $\mathrm{TeV}$ [25] (also [40]). This is interesting for phenomenology and deserves careful study.

The aforementioned separation of the connection into metric and nonmetric contributions is also useful for studies of asymptotic safety. These are using the metric formalism (with a Levi-Civita connection), so they do not take into account nonmetricity effects, etc. Their results could, however, be extended by simply taking into account the new d.o.f. (fields) which are corrections to the Levi-Civita connection. Then asymptotic safety in a nonmetric theory is that of a theory with the Levi-Civita connection plus the dynamical effects of these fields.

\section{CONCLUSIONS}

In this work we studied the effect of Weyl gauge symmetry beyond SM and Einstein gravity, in the context of Weyl conformal geometry. This geometry is of interest since it may play a role in early cosmology or at high scales when effective field theory becomes nearly conformal. To take advantage of its symmetry we used the action expressed in terms of tensors and scalar curvatures of Weyl geometry (instead of their Riemannian expressions) since these are Weyl invariant and covariant, respectively. In this (Weyl) formulation, individual operators in the action are invariant under Weyl gauge symmetry. Then this symmetry and internal gauge symmetries are on an equal footing in the action.

Weyl conformal geometry has a built-in geometric Stueckelberg mass mechanism. By using this Weyl formulation we showed the following: (a) a simple Weyl gauge fixing symmetry transformation easily transforms an action in Weyl geometry directly into an action in Riemannian geometry, due to Stueckelberg breaking of the Weyl gauge symmetry; (b) in this step no fields redefinitions are used, only gauge transformations; (c) no negative kinetic term (ghost) is generated, and the number of d.o.f. is conserved; (d) the Planck scale is an emergent scale where Weyl gauge symmetry is spontaneously broken; hence field values above the Planck scale are natural; and (e) calculations simplify dramatically compared to a Riemannian formulation of this symmetry.

To detail, there is a conservation of the number of dynamical d.o.f. $\left(n_{d f}=3\right)$ in step (a) above, as required for spontaneous breaking: the initial massless gauge field $\omega_{\mu}$ (defining the Weyl connection) absorbs the dilaton (compensator), becomes massive, and then decouples; hence the Weyl connection becomes the Levi-Civita connection. Thus mass generation has a geometric interpretation as a transition from Weyl geometry to the Riemannian one. Note that in the (ungauged) local conformal models, a similar gauge fixing (of the dilaton to a constant VEV) does not conserve $n_{d f}$ when the symmetry breaking takes place, since there is no vector field to "absorb" the Goldstone mode of the symmetry.

Using this idea for the original Weyl quadratic gravity, one finds that this action is immediately transformed by a gauge fixing symmetry transformation, into Einstein-Proca action for the Weyl gauge field plus a (positive) cosmological constant and matter action (if initially present); the Weyl gauge field undergoes a Stueckelberg mechanism. Below its mass $\left(\sim q M_{\text {Planck }}\right)$ this field decouples; hence Einstein gravity is simply a low energy broken phase of Weyl quadratic gravity. No ghost field is present, in contrast with the (ungauged) local conformal models.

Past criticisms of Weyl gravity, related to nonmetricity, assumed the Weyl gauge field to be massless; these criticisms are avoided since such effects induced by the Weyl gauge field are actually strongly suppressed by its mass expected to be high (for $q$ not too small). However, note that current lower bounds on the nonmetricity scale $\left(m_{\omega}\right)$ are low (TeV region). This suggests that the Weyl field could in principle be lighter, if one considers ultraweak values of the coupling $q$, and even act as a dark matter candidate. This would be a "geometric" solution to dark matter since $\omega_{\mu}$ is part of the original Weyl geometry. This is interesting and deserves careful study.

When building Lagrangians with Weyl gauge symmetry, only scalar fields (e.g., the Higgs sector of the SM) couple to the Weyl field $\omega_{\mu}$. Following the same gauge fixing transformation, there exists a "compensating" mechanism for matter scalars with nonminimal couplings to $\tilde{R}$ : if a massless scalar gives a positive (negative) contribution to the generation of the Planck scale, this is "compensated" by a simultaneous negative (positive) mass squared term, i.e., a spontaneous breaking of the symmetry under which it is 
charged. This is due to a dilaton term in the potential induced when "linearizing" the quadratic Weyl scalar curvature term.

Models with Weyl gauge symmetry seem to be allowed by black-hole physics, unlike models with global scale symmetry (e.g., Agravity). Further, models with Weyl gauge symmetry higher dimensional/curvature operators, beyond the quadratic ones of $d=4$ of the Weyl action, are forbidden since they should be suppressed by some high scale not present in the theory and forbidden by this symmetry. Also, the dilaton (compensator) is eaten by the Weyl photon, which becomes massive, so such effective operators could not be suppressed by powers of the dilaton either. This may remain true at the quantum level, assuming quantum calculations respect this symmetry. This is relevant for attempts to prove renormalizability of Weyl gravity action.

Our results may also be of interest to asymptotic safety theories; these are using the metric formalism (Levi-Civita connection) and miss the effects discussed in this work.
However, these studies can be extended to apply here by taking into account the dynamics of the new fields $\left(\omega_{\mu}\right)$ that are corrections to the Levi-Civita connection. So the asymptotic safety in the nonmetric case is that for the Levi-Civita connection plus the additional fields dynamics.

These results indicate that the original Weyl quadratic gravity is physically relevant and its role should be reconsidered, together with its implications for other areas: SM extended with Weyl gauge symmetry/gravity, blackhole physics, cosmology, and supersymmetric version. As stated by Weyl long ago [2]: "The action [...] that was implemented in the previous sections is constituted as [...] a linear combination of $\tilde{R}^{2}$ and $F_{\mu \nu}^{2}$. I believe that one can assert that this action principle implies everything that Einstein's theory has implied up to now, but in the more farreaching questions of cosmology and the constitution of matter, it exhibits a clear superiority. Nevertheless, I do not believe that the laws of nature that are exactly applicable in reality are resolved by it"
[1] H. Weyl, Gravitation und elektrizität, Sitzungsberichte der Königlich Preussischen Akademie der Wissenschaften zu Berlin (1918), p. 465, Einstein's critical comment appended, https://ui.adsabs.harvard.edu/abs/1918SPAW...... $.465 \mathrm{~W} / \mathrm{abstract}$.

[2] H. WeylEine neue Erweiterung der Relativitätstheorie (A new extension of the theory of relativity), Ann. Phys. (Leipzig) 59, 101 (1919).

[3] Raum, Zeit, Materie, Vierte Erweiterte Auflage (Julius Springer, Berlin, 1921) [H. L. Brose, Space-time-matter (Methuen \& Co Ltd, London, 1922)].

[4] For a nice review and references, see E. Scholz, The unexpected resurgence of Weyl geometry in late 20-th century physics, Einstein Stud. 14, 261 (2018); Paving the way for transitions-A case for Weyl geometry, Einstein Stud. 13, 171 (2017); Weyl geometry in late 20th century physics, arXiv:1111.3220.

[5] F. London, Quantum mechanical interpretation of the Weyl theory, Z. Phys. 42, 375 (1927); 5, 253 (1986) (in German and English).

[6] P. A. M. Dirac, Long range forces and broken symmetries, Proc. R. Soc. A 333, 403 (1973).

[7] L. Smolin, Towards a theory of space-time structure at very short distances, Nucl. Phys. B160, 253 (1979).

[8] H. Cheng, The Possible Existence of Weyl's Vector Meson, Phys. Rev. Lett. 61, 2182 (1988).

[9] H. Nishino and S. Rajpoot, Implication of compensator field and local scale invariance in the Standard Model, Phys. Rev. D 79, 125025 (2009).

[10] E. Scholz, Higgs and gravitational scalar fields together induce Weyl gauge, Gen. Relativ. Gravit. 47, 7 (2015).
[11] I. Quiros, Scale invariant theory of gravity and the standard model of particles, arXiv:1401.2643.

[12] Y. Tang and Y. L. Wu, Inflation in gauge theory of gravity with local scaling symmetry and quantum induced symmetry breaking, Phys. Lett. B 784, 163 (2018).

[13] C. T. Hill, Inertial symmetry breaking, arXiv:1803.06994.

[14] M. de Cesare, J. W. Moffat, and M. Sakellariadou, Local conformal symmetry in non-Riemannian geometry and the origin of physical scales, Eur. Phys. J. C 77, 605 (2017).

[15] H. C. Ohanian, Weyl gauge-vector and complex dilaton scalar for conformal symmetry and its breaking, Gen. Relativ. Gravit. 48, 25 (2016).

[16] W. Drechsler and H. Tann, Broken Weyl invariance and the origin of mass, Found. Phys. 29, 1023 (1999).

[17] D. M. Ghilencea and H. M. Lee, Weyl symmetry and its spontaneous breaking in Standard Model and inflation, Phys. Rev. D 99, 115007 (2019).

[18] J. B. Jimenez, L. Heisenberg, and T. S. Koivisto, Cosmology for quadratic gravity in generalized Weyl geometry, J. Cosmol. Astropart. Phys. 04 (2016) 046.

[19] D. M. Ghilencea, Spontaneous breaking of Weyl quadratic gravity to Einstein action and Higgs potential, J. High Energy Phys. 03 (2019) 049.

[20] E. C. G. Stueckelberg, Interaction forces in electrodynamics and in the field theory of nuclear forces, Helv. Phys. Acta 11, 299 (1938).

[21] For a review of the Stueckelberg mechanism, see: H. Ruegg and M. Ruiz-Altaba, The Stueckelberg field, Int. J. Mod. Phys. A 19, 3265 (2004).

[22] R. Percacci, Gravity from a particle physicists' perspective, Proc. Sci., ISFTG2009 (2009) 011; The Higgs phenomenon in quantum gravity, Nucl. Phys. B353, 271 (1991). 
[23] G. 't Hooft, Local conformal symmetry in black holes, Standard Model, and quantum gravity, Int. J. Mod. Phys. D 26, 1730006 (2017); Local conformal symmetry: The missing symmetry component for space and time, arXiv: 1410.6675 .

[24] A. Delhom, G. J. Olmo, and M. Ronco, Observable traces of non-metricity: New constraints on metric-affine gravity, Phys. Lett. B 780, 294 (2018).

[25] Y. Tang and Y. L. Wu, Weyl symmetry inspired inflation and dark matter, arXiv:1904.04493.

[26] D. Gorbunov and V. Rubakov, Introduction to the Theory of the Early Universe (World Scientific, Singapore, 2011).

[27] P. G. Ferreira, C. T. Hill, and G. G. Ross, Inertial spontaneous symmetry breaking and quantum scale invariance, Phys. Rev. D 98, 116012 (2018).

[28] A. A. Starobinsky, A new type of isotropic cosmological models without singularity, Phys. Lett. 91B, 99 (1980); Adv. Ser. Astrophys. Cosmol. 3, 130 (1987).

[29] A. Barnaveli, S. Lucat, and T. Prokopec, Inflation as a spontaneous symmetry breaking of Weyl symmetry, J. Cosmol. Astropart. Phys. 01 (2019) 022.

[30] D. M. Ghilencea, Weyl inflation and an emergent Planck scale, J. High Energy Phys. 10 (2019) 209.

[31] M. Rinaldi and L. Vanzo, Inflation and reheating in theories with spontaneous scale invariance symmetry breaking, Phys. Rev. D 94, 024009 (2016).

[32] P. G. Ferreira, C. T. Hill, and G. G. Ross, Weyl Current, Scale-invariant inflation and Planck scale generation, Phys.
Rev. D 95, 043507 (2017); Scale-independent inflation and hierarchy generation, Phys. Lett. B 763, 174 (2016).

[33] K. Hayashi and T. Kugo, Everything about Weyl's gauge field, Prog. Theor. Phys. 61, 334 (1979).

[34] G. 't Hooft, A class of elementary particle models without any adjustable real parameters, Found. Phys. 41, 1829 (2011); Probing the small distance structure of canonical quantum gravity using the conformal group, arXiv:1009. 0669.

[35] I. Bars, P. Steinhardt, and N. Turok, Local conformal symmetry in physics and cosmology, Phys. Rev. D 89, 043515 (2014).

[36] A. Salvio and A. Strumia, Agravity, J. High Energy Phys. 06 (2014) 080.

[37] R. Kallosh, A. D. Linde, D. A. Linde, and L. Susskind, Gravity and global symmetries, Phys. Rev. D 52, 912 (1995).

[38] F. Englert, C. Truffin, and R. Gastmans, Conformal invariance in quantum gravity, Nucl. Phys. B117, 407 (1976).

[39] M. Shaposhnikov and D. Zenhausern, Quantum scale invariance, cosmological constant and hierarchy problem, Phys. Lett. B 671, 162 (2009); D. M. Ghilencea, Quantum implications of a scale invariant regularization, Phys. Rev. D 97, 075015 (2018); Manifestly scale-invariant regularization and quantum effective operators, Phys. Rev. D 93, 105006 (2016).

40] S. Yaser Ayazi and A. Mohamadnejad, Conformal vector dark matter and strongly first-order electroweak phase transition, J. High Energy Phys. 03 (2019) 181. 